\title{
REMARKS ON GLOBAL ASYMPTOTIC STABILITY OF CERTAIN QUASI-LINEAR DIFFERENTIAL EQUATIONS
}

JAMES S. W. WONG

We consider the following quasi-linear differential equation:

$$
x^{\prime}=A(t) x+f(t, x)
$$

where $A(t)$ is a $n \times n$ matrix with real-valued continuous functions as elements, and $f(t, x)$ is a $n$-vector continuous for $0 \leqq t<\infty,|x|<\infty$. ( $\mid$ denotes the ordinary Euclidean norm.) It is further assumed that the nonlinear term $f(t, x)$ satisfies the following condition:

$$
|f(t, x)| \leqq \lambda(t)|x|,
$$

where $\lambda(t)$ is locally integrable over finite intervals. The purpose of the present note is to give some simple sufficient conditions on $\lambda(t)$ in order that the zero solution of (1) is globally asymptotically stable. Our basic results are the following three simple facts.

THEOREM 1. If there exists a locally integrable function $\mu(t)$ such that the eigenvalues of the symmetric matrix $\frac{1}{2}\left(A(t)+A^{T}(t)\right)$ are less than or equal to $\mu(t)$, and satisfying

$$
\lim _{t \rightarrow \infty} \int_{t_{0}}^{t}(\mu(s)+\lambda(s)) d s=-\infty,
$$

then the zero solution of (1) is globally asymptotically stable.

Proof. Let $V(t, x)=x^{T} x=|x|^{2}$. The total derivative of $V$ with respect to (1) is easily seen to satisfy

$$
V^{\prime}(t, x) \leqq 2(\mu(t)+\lambda(t)) V(t, x) .
$$

Hence a direct integration will yield

$$
V(t, x) \leqq V\left(t_{0}, x_{0}\right) \exp \left\{2 \int_{t}^{t}(\mu(s)+\lambda(s)) d s\right\},
$$

from which the desired conclusion follows.

THEOREM 2. If the fundamental solution matrix $\Phi$ of

$$
x^{\prime}=A(t) x
$$

Received by the editors March 24, 1966. 
satisfies

$$
\left|\Phi(t) \Phi^{-1}(\tau)\right| \leqq k \exp (-\sigma(t-\tau))
$$

for all $t, \tau \geqq 0$, where $k$, and $\sigma$ are positive constants,

$$
\lim _{t \rightarrow \infty} \int_{t_{0}}^{t}\left(\lambda(t)-\frac{\sigma}{k^{2}}\right) d t=-\infty,
$$

then the zero solution of (1) is globally asymptotically stable.

Proof. According to a result of Malkin [1], if the solution of (4) satisfy (5), then the following quadratic form

$$
V(t, x)=x^{T} C(t) x=\int_{t}^{\infty}\left|\Phi(\tau) \Phi^{-1}(t) x\right|^{2} d \tau .
$$

with some positive definite symmetric matrix $C(t)$, is a Lyapunov function for (4) whose total derivative with respect to (4) is

$$
V^{\prime}(t, x)=x^{T}\left(A^{T} C(t)+C(t) A+C^{\prime}(t)\right) x=-|x|^{2} .
$$

It is easily computed that the total derivative $V^{\prime}(t, x)$ with respect to (1) is

$$
V^{\prime}(t, x)=x^{T}\left(A^{T} C(t)+C(t) A+C^{\prime}(t)\right) x+f^{T} C(t) x+x^{T} C(t) f
$$

Since $C(t)$ is positive definite and symmetric, there exists $C_{1}>0$, such that $V(t, x) \geqq C_{1}|x|^{2}$, and we have from (2) that

$$
f^{T} C(t) x+x^{T} C(t) x \leqq 2 \lambda(t) V(t, x) .
$$

Estimating $V(t, x)$ by (5), we obtain for (8) the following inequality:

$$
\begin{aligned}
V^{\prime}(t, x) & \leqq-|x|^{2}+\frac{\lambda(t) k^{2}}{\sigma}|x|^{2} \\
& =\frac{k^{2}}{\sigma}\left(\lambda(t)-\frac{\sigma}{k^{2}}\right)|x|^{2} \\
& \leqq \frac{k^{2}}{\sigma C_{1}}\left(\lambda(t)-\frac{\sigma}{k^{2}}\right) V(t, x),
\end{aligned}
$$

from which the desired conclusion follows by a direct in tegration.

We now specialize (1) to the case when $A(t)$ is a constant stable matrix, i.e. all eigenvalues of $A$ are of the form: $\lambda_{k}=-\sigma_{k}+i \tau_{k}\left(\sigma_{k}>0\right)$. Denote the multiplicity of $\lambda_{k}$ by $m_{k}$, and let $T=\left(t_{i j}\right)$ be the nonsingular matrix such that $T^{-1} A T=J$ is the Jordan canonical form of $A$. 


$$
|T|=\left(\sum_{i, j=1}^{n}\left|t_{i j}\right|^{2}\right)^{1 / 2} \text {. }
$$

If

$$
\lim _{t \rightarrow \infty} \int_{t_{0}}^{t}\left(\lambda(s)-\frac{\bar{\sigma}}{|T|\left|T^{-1}\right|}\right) d s=-\infty,
$$

where $\bar{\sigma}<\min _{k} \sigma_{k}$, then the zero solution of (1) is globally asymptotically stable.

Proof. We first transform (1) into the following equation by putting $y=T^{-1} x$,

$$
y^{\prime}=J y+T^{-1} f(t, T y) .
$$

Let $\Psi$ be the fundamental matrix solution of $y^{\prime}=J y$ satisfying $\Psi(0)$ $=I$. It is readily seen that $\Psi$ satisfies $\left|\Psi(t) \Psi^{-1}(\tau)\right| \leqq \exp [-\bar{\sigma}(t-\tau)]$ and $|f(t, T y)| \leqq \lambda(t)|T||y|$. Applying Theorem 2 to (10), we obtain the desired conclusion.

REMARK 1. Theorem 1 is useful in providing sufficient conditions for asymptotic stability of (1) when $A(t)$ is symmetric. Consider the following system:

$$
\left(\begin{array}{l}
x_{1} \\
x_{2}
\end{array}\right)^{\prime}=\left(\begin{array}{rr}
-\frac{1}{t} & \frac{1}{2 t} \\
\frac{1}{2 t} & -\frac{1}{t}
\end{array}\right)\left(\begin{array}{l}
x_{1} \\
x_{2}
\end{array}\right)+e^{-t}\left(\begin{array}{l}
x_{1} \\
x_{2}
\end{array}\right) .
$$

In this case, we have $\mu(t)=-1 / 2 t$ and $\lambda(t)=e^{-t}$, hence (3) is clearly satisfied. On the other hand, the eigenvalues of $A(t)$ are not bounded by a negative constant and so the classical stability theorems do not apply.

REMARK 2. We note that for a stable matrix $A, \frac{1}{2}\left(A+A^{T}\right)$ may be unstable. Consider

$$
A=\left(\begin{array}{ll}
2 & 3 \\
1 & 2
\end{array}\right)
$$

Consequently, Theorem 3 may not be considered as a special case of Theorem 1.

REMARK 3. Theorems 2 and 3 generalize a result of Levinson [2] on the asymptotic stability of (1) when $f(t, x)$ is linear in which it is required that $\int^{\infty} \lambda(t) d t<\infty$ or $\lim _{t \rightarrow \infty} \lambda(t)=0$. The above result [2] has recently been extended to systems of the form (1) with an integrable 
forcing term. (See Brauer [3].) With appropriate modifications, our results may be stated for this slightly more general case as well.

REMARK 4. Theorem 3 generalizes a recent result of Burton [4], ${ }^{1}$ in which it is shown that if $\lambda(t) \leqq k$ for $t \geqq 0$, where $k<\bar{\sigma} /|T|\left|T^{-1}\right|$ for some appropriate choice of $\bar{\sigma}$, then the zero solution of (1) is globally asymptotically stable.

REMARK 5. We may also use Theorem 3 to discuss a stability estimate recently given by Hochstadt [5] in case $f(t, x)=\mu B(t) x$, where $B(t)$ is a $n \times n$ matrix which is periodic with period $2 \pi$ and has an absolutely convergent Fourier series. In case $A$ is given in its Jordan canonical form and $\sup _{t}|B(t)|=\beta$, Hochstadt's estimate in terms of our terminology is

$$
\mu \beta<\min _{\boldsymbol{k}} \frac{\sigma_{k}^{m_{k}}}{1+\sigma_{k}+\cdots+\sigma_{k}^{m_{k-1}}} .
$$

In this case, the above results will yield $\mu \beta<\min _{k} \sigma_{k}$.

REMARK 6. All three theorems above are best possible in the sense that if (3), (6) or (9) fails to hold then there exists system with solutions not tending to zero. Consider the first-order equation $x^{\prime}(t)$ $+\lambda(t) x(t)=0$ with $\int^{\infty} \lambda(s) d s \leqq M$. In this case, $A(t) \equiv 0$, and all three conditions (3), (6), and (9) do not hold. Any solution $x(t)$ with $x\left(t_{0}\right)$ $\neq 0$ does not tend to zero, i.e.

$$
x(t)=x\left(t_{0}\right) \exp \left(-\int^{t} \lambda(s) d s\right) \geqq x\left(t_{0}\right) e^{-M}>0 .
$$

REMARK 7. In contrast to conditions on $\lambda(t)$ as imposed in [4], [5] for asymptotic stability, our results ensure global asymptotic stability.

\section{REFERENCES}

1. I. G. Malkin, On the construction of Liapunov functions for systems of linear equations, Akad. Nauk SSR Prikl. Mat. Meh. 16 (1952), 239-242.

2. N. Levinson, The asymptotic hehaviour of a system of linear differential equations, Amer. J. Math. 68 (1946), 1-6.

3. F. Brauer, Non-linear differential equations with forcing terms, Proc. Amer. Math. Soc. 15 (1964), 758-765.

4. T. A. Burton, On a theorem of Brauer, Proc. Amer. Math. Soc. 17 (1966), $325-326$.

5. H. Hochstadt, A stability estimate for differential equations with periodic coefficients, Arch. Math. 15 (1965), 318-320.

University of Alberta, Edmonton, Alberta, Canada.

1 The author wishes to thank T. A. Burton for a copy of reference [4] prior to its publication. 